\title{
Thermodynamics of ionic microgels
}

\author{
Yan Levin, ${ }^{1}$ Alexandre Diehl, ${ }^{2}$ A. Fernández-Nieves ${ }^{3}$ and A. Fernández-Barbero ${ }^{3}$ \\ ${ }^{1}$ Instituto de Física, Universidade Federal do Rio Grande do Sul, Caixa Postal 15051, CEP 91501-970 Porto Alegre, \\ Rio Grande do Sul, Brazil \\ ${ }^{2}$ Departamento de Física, Universidade Federal do Ceará, Caixa Postal 6030, CEP 60455-760 Fortaleza, Ceara, Brazil \\ ${ }^{3}$ Group of Complex Fluids Physics, Department of Applied Physics, University of Almeria, 04120 Almería, Spain
}

(Received 12 September 2001; published 7 March 2002)

\begin{abstract}
We present a theory of dilute aqueous suspensions of microgel particles. It is found that as the number of charged monomers in the polymer network composing mesoscopic gel increases, the particles undergo a swelling transition. Depending on the hydrophobicity of the polymer, this transition can be either continuous or discontinuous. Furthermore, similar to charge stabilized colloidal particles, we find that the electrophoretic mobility of the microgel is controlled by an effective charge. Unlike the colloids, however, for which the effective charge grows asymptotically with the logarithm of the bare charge, the effective charge of an ionic microgel scales as $Z_{\text {eff }} \sim Z^{0.5}$. The findings are in good agreement with the experimental measurements.
\end{abstract}

DOI: 10.1103/PhysRevE.65.036143

PACS number(s): 64.60.-i, 05.70.Fh

\section{INTRODUCTION}

Since the early work of Flory [1] in the 1940s and 1950s our understanding of gelation has steadily improved. In medicine, biology, and industry in general, gels have become quite ubiquitous. Over the years, a particular class of gels called polyelectrolyte gels $[2,3]$ has attracted a lot of attention due to the vast potential of their industrial application. A polyelectrolyte gel consists of an aqueous solution of a crosslinked polymer network. Some of the monomers of the polymer have an acidic (basic) nature. Thus, the volume spanned by the gel is strongly dependent on the degree of ionization of the individual monomers. If a sufficiently large fraction of monomers is ionized, the electrostatic repulsion and the osmotic pressure of ions can swell the network, increasing its volume by some orders of magnitude. The strong dependence of the network's volume on the $p \mathrm{H}$ of the surrounding solvent makes polyelectrolyte gels an ideal candidate for the design of artificial muscles [4]. This fact was already noticed in the early days of polymer theory by Katchalsky [2].

Recently gel particles with colloidal dimensions have become a focus of considerable scientific research [5]. Strong size dependence of these particles on $p \mathrm{H}$ provides for some very interesting reological and viscoelastic properties [6]. The possibility of using microgels as vehicles for drug delivery [7], thus complimenting the traditional lyposome based systems, has also not been overlooked. In addition, similarity between microgels and the star polyelectrolytes, which have recently been attracting some attention $[8,9]$, might also prove to be useful.

Unfortunately, unlike for the macroscopic gel networks, the thermodynamics of microgels has not been fully developed. The difficulty lies in the long-ranged nature of the Coulomb force. The same problem has hindered understanding the phase structure of charge stabilized colloidal suspensions. Thus, for nearly two decades an argument has been raging about whether a charged colloidal suspension is stable against a fluid-fluid phase separation in a low salinity solvent [10-14]. Although the issue has not been fully resolved, there is mounting evidence that the answer to this question is indeed affirmative $[12,15,17,18]$.

In this paper, we shall construct a thermodynamic theory of suspensions composed of microgel particles. The results of the theory will be compared with the experimental measurements, allowing one to judge the extent of its validity.

The outline of the paper is as follows. Section II will present a theory employed for the thermodynamic description of mesoscopic gels. In Sec. III, the experimental system and methods used to test the theory will be described. In Sec. IV, we will compare the theoretical predictions with the experimental measurements.

\section{THE MODEL}

To simplify the calculations we shall adopt a mean-field picture in which each particle of a suspension is confined to a spherical Wigner-Seitz cell, whose radius $R_{2}$ is determined by the concentration of microgel particles. Although, this approach will not allow us to study the full phase diagram, it should be sufficient to answer some key questions related to the effective charge of the microgel particles as well as their swelling transitions.

Thus, we shall represent the microgel particle as a spherical cross-linked polymer network of radius of gyration $R_{1}$ $\ll R_{2}$, composed of $N_{2}$ chains, as shown in Fig. 1. Each chain carries $m$ monomers each of molecular volume $v_{2}$, of which a fraction $f$ is assumed to be of strong electrolyte nature. Thus, in aqueous solution $\left(m f N_{2}\right)$ ionizable groups will dissociate, leaving behind charged monomers. The dissociated counterions can either leave the interior of the microgel particle, in which case we can say that they are "free," or they can stay in the interior of the microgel, in that case we shall call them "condensed."

For simplicity, the $N_{1}$ particles of solvent inside the Wigner-Seitz cell will be assumed to have the same molecular volume $v_{1}$ as the counterions. Since we will be concerned with the incompressible solvents, such as water, the number of molecules of solvent $N_{1}$ is given by 


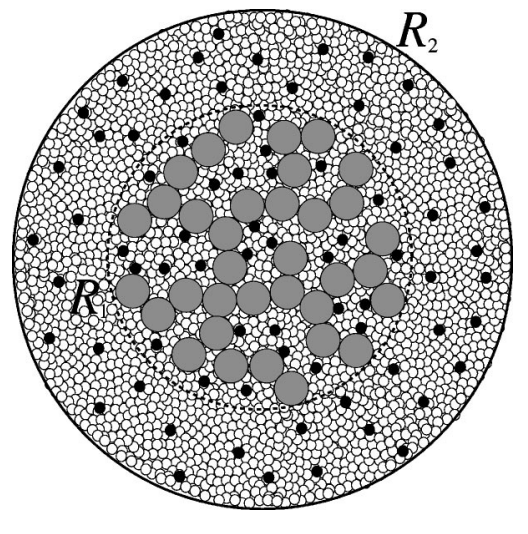

FIG. 1. Schematic depiction of the model: a spherical microgel particle of radius $R_{1}$, composed of $m N_{2}$ monomers (large dark spheres), is immersed into a solution of water molecules (small hollow spheres) and counterions (small black spheres) inside a spherical Wigner-Seitz cell of radius $R_{2}$.

$$
N_{1}=\frac{1}{v_{1}}\left[\frac{4}{3} \pi R_{2}^{3}-v_{2} m N_{2}\left(1+f \frac{v_{1}}{v_{2}}\right)\right]
$$

Let us now define $N_{1}^{\mathrm{i}}=x N_{1}$ and $N_{3}^{\mathrm{i}}=y m f N_{2}$ as the number of solvent molecules and the counterions, respectively, which are inside the microgel. The $x$ and $y$ are the relative fractions.

Outside the microgel there are then $N_{1}^{0}=(1-x) N_{1}$ molecules of solvent and $N_{3}^{0}=(1-y) m f N_{2}$ counterions. Note that the counterions and the solvent molecules are free to move throughout the whole volume $V=4 \pi R_{2}^{3} / 3$ of the Wigner-Seitz cell (Fig. 1). The fraction of particles that remains inside the microgel is determined by the equilibrium condition of the minimum of Helmholtz free energy.

The Helmholtz free energy for the cell can be written as a sum of the "inside" and the "outside" contributions, $\beta F$ $=\beta F^{\text {in }}+\beta F^{\text {out }}$. Unfortunately, there is no exact way to obtain these free energies. We shall, therefore, approximate them by a sum of the most relevant contributions: entropic, elastic, solute-solvent, and electrostatic. We find that $\beta F^{\text {in }}$ $=\beta F_{\text {mix }}+\beta F_{\text {elas }}+\beta F_{\text {sol-gel }}+\beta F_{\text {elet }}$. Since we are mostly concerned with a very dilute suspensions, we shall neglect the electrostatic interactions between the free counterions and the microgel, so that $\beta F^{\text {out }}$, is a purely entropic free energy of mixing.

Free energy of mixing. Inside the microgel there is a mixture of polymer, counterions, and solvent molecules. The entropic contribution to the free energy can be expressed using the Flory-Huggins theory [1] as,

$$
\beta F_{\operatorname{mix}}=N_{1}^{i} \ln \phi_{1}^{i}+N_{3}^{i} \ln \phi_{3}^{i}
$$

where

$$
\phi_{1}^{i}=\frac{v_{1} N_{1}^{i}}{m v_{2} N_{2}+v_{1} N_{1}^{i}+v_{1} N_{3}^{i}}
$$

is the volume fraction of solvent inside the microgel, and

$$
\phi_{3}^{i}=\frac{v_{1} N_{3}^{i}}{m v_{2} N_{2}+v_{1} N_{1}^{i}+v_{1} N_{3}^{i}}
$$

is the volume fraction of counterions inside the same region.

In the outside region, the entropic contribution is

$$
\beta F^{\text {out }}=N_{1}^{0} \ln \phi_{1}^{0}+N_{3}^{0} \ln \phi_{3}^{0},
$$

where

$$
\phi_{1}^{0}=\frac{N_{1}^{0}}{N_{1}^{0}+N_{3}^{0}} \quad \text { and } \quad \phi_{3}^{0}=\frac{N_{3}^{0}}{N_{1}^{0}+N_{3}^{0}}
$$

are the volume fractions of solvent and counterions outside the microgel, respectively. Note that since we are dealing with a dilute suspension and are neglecting the electrostatic interactions in the outside region, this is the only contribution to $F^{\text {out }}$.

Elastic free energy. According to the Flory's affine network theory [1], the elastic free energy due to elongation and contraction of the $N_{2}$ chains of the microgel is

$$
\beta F_{\text {elas }}=\frac{3}{2} N_{2}\left[\alpha^{2}-1-\ln \alpha\right]
$$

where $\alpha$ is the expansion factor of the microgel compared to its dry state,

$$
\alpha^{3}=\frac{m v_{2} N_{2}+v_{1} N_{1}^{i}+v_{1} N_{3}^{i}}{m v_{2} N_{2}+m v_{1} f N_{2}}=\frac{1}{\phi_{2}\left(1+f v_{1} / v_{2}\right)} .
$$

Here, $\phi_{2}$ is the volume fraction occupied by the monomers of the polymer network inside the region $r \leqslant R_{1}$,

$$
\phi_{2}=\frac{m v_{2} N_{2}}{m v_{2} N_{2}+v_{1} N_{1}^{i}+v_{1} N_{3}^{i}} .
$$

Polymer-solvent free energy. Interaction between the monomers of microgel and the molecules of solvent is taken into account through the

$$
\beta F_{\text {sol-gel }}=\chi m N_{2} \phi_{1}^{i}
$$

contribution to the total free energy. Here, $\chi$ is the Flory polymer-solvent interaction parameter.

Electrostatic free energy. To calculate the electrostatic contribution to the free energy, let us suppose that the counterions are distributed uniformly inside the microgel. The effective gel charge can then be written as

$$
Q \equiv Z_{\mathrm{eff}} q=q\left(m f N_{2}-N_{3}^{i}\right)
$$

The electrostatic energy of a uniformly charged sphere of radius $R_{1}$ can be obtained as

$$
F_{\text {elet }}=\int_{0}^{R_{1}} \frac{\left(\rho_{q} 4 \pi r^{3} / 3\right) \rho_{q} 4 \pi r^{2}}{\epsilon r} d r
$$


where $\epsilon$ is the dielectric constant inside the microgel, which we shall assume to be equivalent to that of pure solvent (water). The $\rho_{q}=Q /\left(4 \pi R_{1}^{3} / 3\right)$ is the effective charge density inside $R_{1}$. The electrostatic contribution to the free energy is then given by

$$
\beta F_{\text {elet }}=\frac{3}{5} \frac{\lambda_{B}}{R_{1}}\left(m f N_{2}-N_{3}^{i}\right)^{2},
$$

where $\lambda_{B}=\beta q^{2} / \epsilon=7.2 \AA$ is the Bjerrum length for water and

$$
R_{1}=\left[\frac{3}{4 \pi}\left(m v_{2} N_{2}+v_{1} N_{1}^{i}+v_{1} N_{3}^{i}\right)\right]^{1 / 3}
$$

is the radius of microgel. Note that this is a purely mean-field contribution to the electrostatic free energy. Nowhere do we account for the correlations between the condensed counterions and charged monomers. In a more refined calculation these contributions to the total free energy will have to be included.

For a fixed volume and number of particles, the equilibrium distribution of counterions and solvent between the inside and the outside regions is determined by the minimum of the total Helmholtz free energy, $\delta\left(\beta F^{\text {in }}+\beta F^{\text {out }}\right)=0$. Equivalently, in terms of variables $x$ and $y$ this reduces to

$$
\frac{\partial \beta F}{\partial x}=0, \text { and } \quad \frac{\partial \beta F}{\partial y}=0 .
$$

The equations for the equilibrium fractions $x$ and $y$ that result from Eq. (15) are

$$
\begin{aligned}
N_{1} \phi_{2} & +N_{1} \ln \phi_{1}^{i}+N_{1}\left(1+y f \frac{v_{1}}{v_{2}}\right) \frac{v_{1}}{v_{2}} \chi \phi_{2}^{2} \\
+ & \frac{v_{1}}{v_{2}} \frac{N_{1}}{m}\left[\frac{\phi_{2}^{1 / 3}}{\left(1+f \frac{v_{1}}{v_{2}}\right)^{2 / 3}}-\frac{\phi_{2}}{2}\right] \\
& -\frac{3 \lambda_{B} v_{1} N_{1}\left(m f N_{2}\right)^{2}(1-y)^{2}}{20 \pi R_{1}^{4}}-N_{1} \ln \phi_{1}^{0}=0,
\end{aligned}
$$

and

$$
\begin{aligned}
m f N_{2} & \phi_{2}+m f N_{2} \ln \phi_{3}^{\mathrm{i}}-f x N_{1}\left(\frac{v_{1}}{v_{2}}\right)^{2} \chi \phi_{2}^{2} \\
+ & +\frac{v_{1}}{v_{2}} f N_{2}\left[\frac{\phi_{2}^{1 / 3}}{\left(1+f \frac{v_{1}}{v_{2}}\right)^{2 / 3}}-\frac{\phi_{2}}{2}\right] \\
& -\frac{3 \lambda_{B} v_{1}\left(m f N_{2}\right)^{3}(1-y)^{2}}{20 \pi R_{1}^{4}}-\frac{6 \lambda_{B}}{5 R_{1}}\left(m f N_{2}\right)^{2}(1-y) \\
& -m f N_{2} \ln \phi_{3}^{0}=0
\end{aligned}
$$

If all the counterions were to be confined to the microgel (condensed), so that $y=1$, and $Z_{\text {eff }}=0$ and $\phi_{1}^{0}=1$, Eq. (16) would reduce to an equilibrium condition similar to the one previously studied by Fernández-Nieves et al. [5], with the rescaled Flory polymer-solvent parameter $\chi_{R}=\left(v_{1} / v_{2}\right) \chi$. In reality, however, the counterions are free to leave the microgel, and one is forced to study the more general equations. (16) and (17).

\section{EXPERIMENTAL DETAILS}

The synthesis of the system to be used to verify some of the theoretical predictions of this paper has been described elsewhere [19]. The microgel particles are based on poly(2-vinylpyridine) (2VP), crosslinked with divinylbenzene (DVB, 0.25 wt. \%). The initiator used was 2,2'-azobis(2-amidinopropane)dihydrochloride (V50, Wako). Both the 2VP and DVB groups are expected to be uniformly distributed throughout the bulk of the particles. The number of chains inside each microgel particle is $N_{2}=3 \times 10^{5}[5,19]$. The number of monomers (charged or not) in the microgel is approximately $3 \times 10^{7}$, which implies the number of monomers per chain equal to $m=100$.

Two chemical groups are able to confer charge to the colloidal particles: (i) amidinium groups arising from the initiator, located essentially at the periphery of the particles, and (ii) the constituent monomer $2 \mathrm{VP}$. The presence of surface charge is necessary in order to keep the system stable for deswollen particle states. The $p K_{a}$ of each group is 10 and 5, respectively [20]. The surface and bulk charges were determined by titration and are both controlled through the medium $p \mathrm{H}$ [5]. However, when the ionization of the network polymer groups starts, all surface groups are already protonated, thus $Z^{(s)}$ (the surface charge) remains unaltered while $Z$ is being changed [5]. This is an important fact since it allows us to verify predictions concerning the effective charge $Z_{\text {eff }}$, without having to superimpose the influence of $Z^{(s)}$

Transmission electron microscopy (TEM) showed that the collapsed diameter of the microgel is $2 R_{1}^{\mathrm{TEM}}=(2050$ $\pm 80) \AA[19]$. The ionization of the polymer network is accomplished by decreasing the medium $p \mathrm{H}$ and results in gel swelling. Electrophoretic mobility measurements will allow us to access the effective bulk charge $Z_{\text {eff }}$ as a function of the bare charge $Z$. The data were obtained by Doppler light scattering using a Zetamaster- $S$ (Malvern Instruments), working with a $6328 \AA \mathrm{He}-\mathrm{Ne}$ laser. All measurements were carried out in dilute regime $\left(\phi \sim 10^{-5}\right)$, in order to avoid undesirable interactions between particles and multiple scattering. The medium $p \mathrm{H}$ was fixed by the addition of $\mathrm{HCl}$ or $\mathrm{NaOH}$ while the background electrolyte was fixed to $1 \mathrm{mM}$ by using $\mathrm{NaCl}$. The temperature was set to $(25.0 \pm 0.1){ }^{\circ} \mathrm{C}$ in all the experiments.

\section{RESULTS AND DISCUSSION}

\section{A. Theoretical predictions}

The equilibrium configurations for the microgel are given by the solutions of coupled Eqs. (16) and (17). The radius $R_{2}$ 


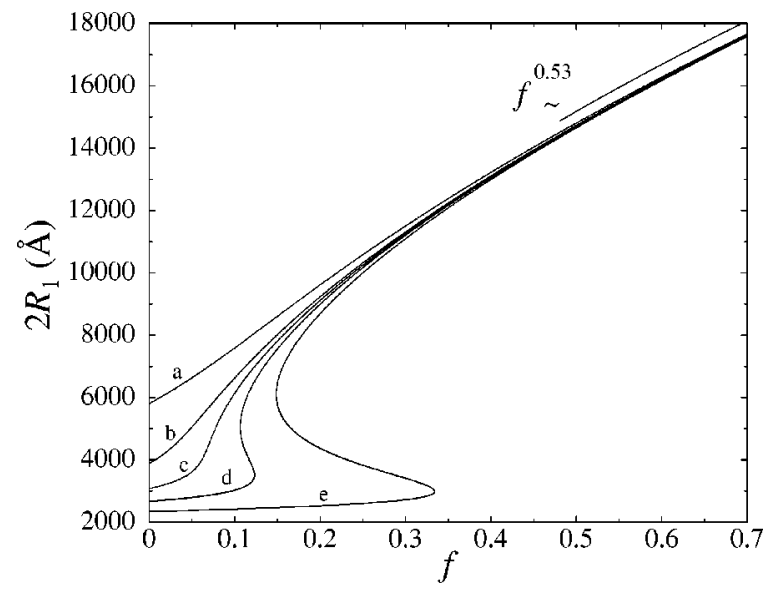

FIG. 2. Microgel diameter $2 R_{1}$ vs fraction $f$ of charged monomers for different values of rescaled polymer-solvent parameter $\chi_{R}$ : (a) 0.1, (b) 0.5, (c) 0.6, (d) 0.7, and (e) 0.9. For small $\chi_{R}$, the microgel swells continuously with the increase of bare charge, while for larger $\chi_{R}$ a discontinuous transition from a collapsed to an extended state is observed.

is fixed by the volume fraction of the microgel particles. Using $R_{1}^{\mathrm{TEM}}$ as a reference and a particle volume fraction of $10^{-5}$, we obtain $R_{2} \approx 1 \times 10^{5} \AA$. Finally, we use $v_{1}$ $=30 \AA^{3}$ for the molecular volume of solvent and counterions, and $v_{2}=140 \AA^{3}$ for the molecular volume of monomers. The value of $v_{1}$ is obtained by considering the density of water $1 \mathrm{~g} / \mathrm{cm}^{3}$, while the value of $v_{2}$ is determined from the $R_{1}^{\mathrm{TEM}}$ and the estimate of the number of monomers per microgel particle, discussed earlier.

Figure 2 shows the dependence of microgel diameter on the fraction of charged monomers $f$ for different values of the rescaled polymer-solvent parameter $\chi_{R}$. For low charge, the microgel diameter is determined mainly by the polymersolvent interactions. For small values of $\chi_{R}$ the contact between solvent and monomers does not cost much energy and a large number of solvent molecules are found inside the radius $R_{1}$. Hence, the microgel is in an extended configuration. As $\chi_{R}$ increases the monomer-solvent interaction becomes more unfavorable, forcing the solvent molecules to leave the microgel. For $\chi_{R}=0.9$ the deswollen state of a microgel particle has a diameter of approximately $2350 \AA$ close to the value found by TEM (Fig. 2). Increasing the fraction of charged monomers leads to swelling of the microgel. For small values of $\chi_{R}$ the particle diameter increases continuously with $f$, while for larger $\chi_{R}$ a van der Waals-like loop appears, indicating the existence of a first-order transition between the two equilibrium sizes (Fig. 2). Since no dependence of $R_{1}$ on $\chi_{R}$ is found for large $f$, the size of a highly ionized gel is controlled purely by the electrostatic and elastic forces, and is independent of the particular nature of colloidal gel.

The dependence of the microgel size on the effective charge $Z_{\text {eff }}=m f N_{2}(1-y)$ is shown in Fig. 3. For a small fraction of charged monomers $f$, the particle diameter is quite insensitive to the effective charge, since in this regime the particle size is controlled by the polymer-solvent interactions. However, for larger $f$ values, an almost linear depen-

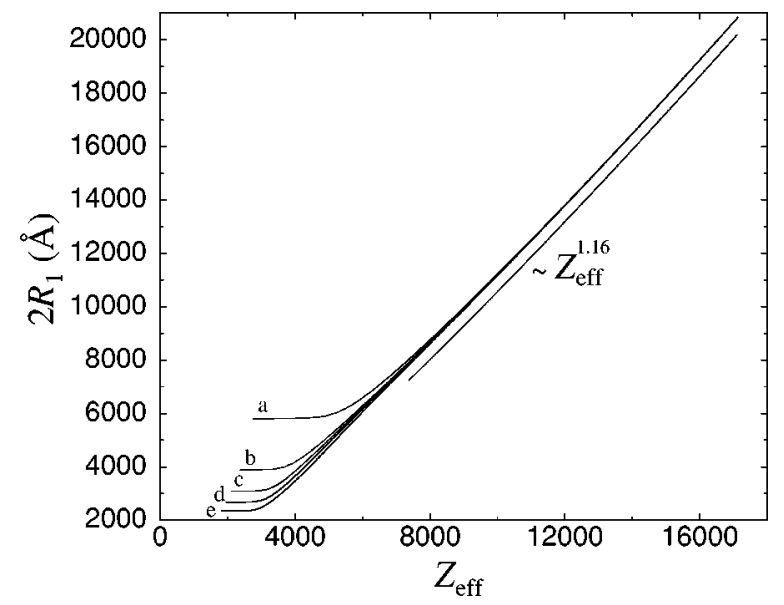

FIG. 3. Microgel diameter as a function of the effective charge, for different values of $\chi_{R}$ (see Fig. 2 for reference). Note that for weakly charged particles the diameter is independent of the effective charge, while for strongly charged particles the diameter is almost linearly proportional to the effective charge.

dence of the particle diameter on the effective charge is found.

In Fig. 4, we show another interesting aspect of the microgel problem. For strongly charged particles the effective charge of a microgel scales with the bare charge as $Z^{0.46}$. This dependence is in complete contrast to what was found for strongly charged colloidal particles. In that case the effective charge was found to grow as a logarithm of the bare charge $[12,15,16]$.

\section{B. Experimental verification of the theory}

In order to test the validity of the asymptotic behavior $Z_{\text {eff }} \sim Z^{0.5}$, electrophoretic mobility measurements were undertaken. Recall that the theoretical prediction $2 R_{1} \sim Z_{\text {eff }}$ was indirectly verified in Ref. [5]. In that work it was experimentally shown that $2 R_{1} \sim Z^{0.5}$. Since the present theory predicts a square root dependence between effective and bare charges (see Fig. 4), it follows that the size scales linearly with $Z_{\text {eff }}$.

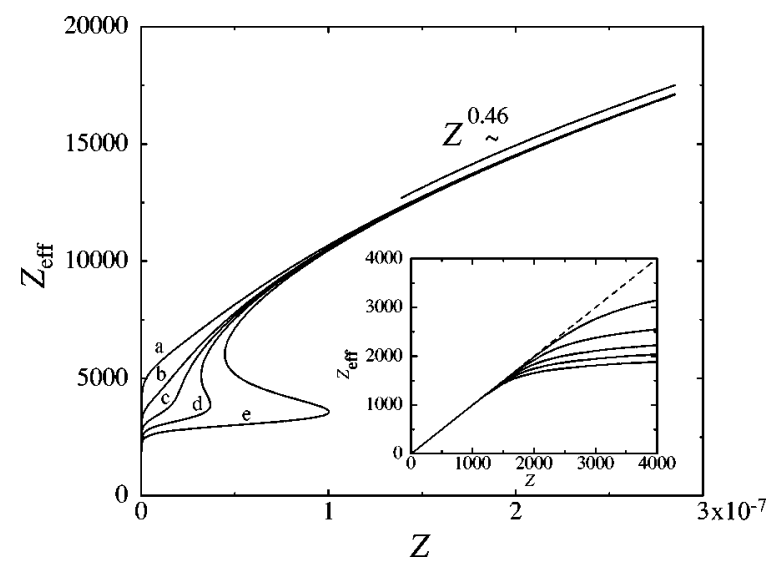

FIG. 4. Effective charge vs bare charge of a microgel particle for the polymer-solvent parameters shown in Fig. 2. The inset shows the absence of charge renormalization for weakly charged microgels, $Z_{\text {eff }}=Z$. 
Assuming that the electrostatic potential at equilibrium satisfies the superposition principle, implying that it can be written as a sum of the surface and bulk contributions, and employing the linearized Poisson-Boltzmann equation, electrophoretic mobility can be written as [21]

$$
\mu=\mu_{s}+\mu_{b}=Z_{\text {eff }}^{(\mathrm{s})} f\left(\kappa, \lambda, R_{1}\right)+Z_{\text {eff }} g\left(\kappa, \lambda, R_{1}\right),
$$

where $\mu_{s}$ is the electrophoretic mobility arising from the surface charge, while $\mu_{b}$ is the electrophoretic mobility due to bulk charges. $Z_{\text {eff }}^{(\mathrm{s})}$ and $Z_{\text {eff }}$ stand for the effective surface and bulk charges, respectively, $\kappa$ is the inverse of the Debye length, and $\lambda=(\gamma / \eta)^{1 / 2}$ is defined in terms of the friction coefficient $\gamma$ and the viscosity $\eta$. The functions $f$ and $g$ are given by

$$
\begin{aligned}
f\left(\kappa, \lambda, R_{1}\right)= & \frac{1}{4 \pi R_{1}^{2} \eta \lambda^{2}}\left[\frac{3}{R_{1}}+\frac{2}{R_{1}}\left(1-\frac{\lambda R_{1}}{3}\right.\right. \\
& \times \frac{\sinh \left(\lambda R_{1}\right)}{\left.\left.\cosh \left(\lambda R_{1}\right)-\frac{\sinh \left(\lambda R_{1}\right)}{\lambda R_{1}}\right)+\frac{2 R_{1} \lambda^{2}}{3\left(1+\kappa R_{1}\right)}\right]}
\end{aligned}
$$

and

$$
\begin{aligned}
g\left(\kappa, \lambda, R_{1}\right)= & \frac{3}{4 \pi R_{1}^{3} \eta \lambda^{2}}\left[1+\frac{1}{3}\left(\frac{\lambda}{\kappa}\right)^{2}\left(1+e^{-2 \kappa R_{1}}\right.\right. \\
& \left.\left.-\frac{1-e^{-2 \kappa R_{1}}}{\kappa R_{1}}\right)+h\left(\kappa, \lambda, R_{1}\right)\right]
\end{aligned}
$$

where

$$
\begin{aligned}
h\left(\kappa, \lambda, R_{1}\right)= & \frac{1}{3} \frac{\lambda^{2}}{\kappa^{2}} \frac{1+1 / \kappa R_{1}}{\lambda^{2} / \kappa^{2}-1} \\
& \times\left(\frac{\lambda}{\kappa} \frac{1+e^{-2 \kappa R_{1}}-\left(1-e^{-2 \kappa R_{1}}\right) / \kappa R_{1}}{\left(1+e^{-2 \lambda R_{1}}\right) /\left(1-e^{-2 \lambda R_{1}}\right)-1 / \lambda R_{1}}\right. \\
& \left.-1+e^{-2 \kappa R_{1}}\right) .
\end{aligned}
$$

As can be seen from Eq. (18), the electrophoretic mobility depends on the effective charges, $Z_{\text {eff }}^{(\mathrm{s})}$ and $Z_{\text {eff }}$. Thus, when one of the two is kept constant, the electrophoretic mobility is directly dependent on the other effective charge variation. In the experiment considered here, $Z_{\text {eff }}^{(\mathrm{s})}$ is kept constant so that the electrophoretic mobility is a function of $Z_{\text {eff }}$ only. Dividing Eq. (18) by $\mu_{s}$, we can write

$$
\mu^{*}=\frac{\mu}{\mu_{s}}=1+Z_{\mathrm{eff}} \frac{g\left(\lambda, \kappa, R_{1}\right)}{\mu_{s}} .
$$

If $g\left(\kappa, \lambda, R_{1}\right) / f\left(\kappa, \lambda, R_{1}\right)$ does not vary with the particle swelling, which is induced by increasing $Z, \mu^{*}$ is a linear

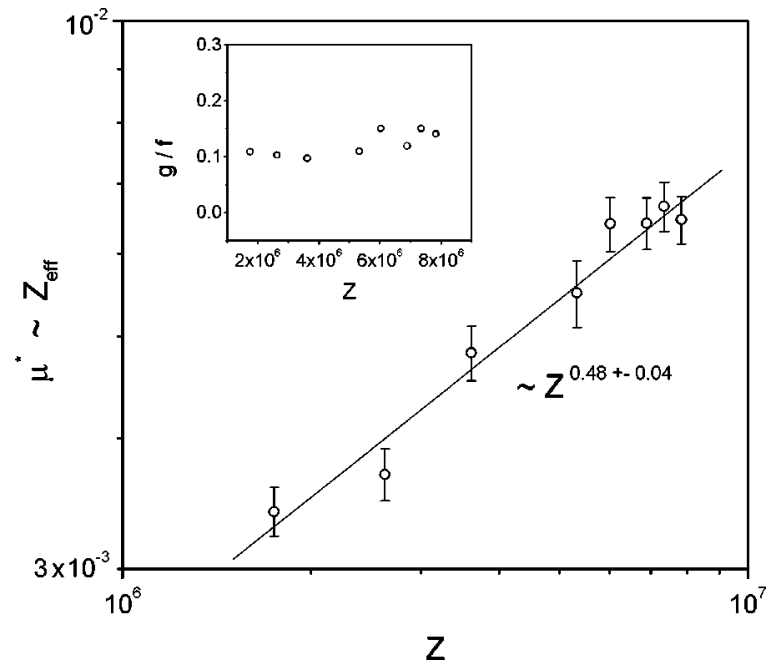

FIG. 5. Experimental verification of the theoretical prediction $Z_{\text {eff }} \sim Z^{0.5}$. The effective charge is a linear function of $\mu^{*}$, which is obtained from the experimental $\mu$ measurements.

function of $Z_{\text {eff }}$ and can be used to test the theoretical prediction of the asymptotic behavior $Z_{\text {eff }} \sim Z^{0.5}$. The fact that the ratio $g\left(\kappa, \lambda, R_{1}\right) / f\left(\kappa, \lambda, R_{1}\right)$ is almost constant, independent of $Z$, can be seen from the inset of Fig. 5. In order to compute the functions $f$ and $g$, the friction exerted on the liquid flow by the polymer chains needs to be modeled. This frictional force is controlled by the friction coefficient $\gamma$. Treating the gel as a spherical cloud of homogeneously distributed beads and using the theory of Brinkman [22], together with the modifications proposed in Ref. [23], the friction coefficient can be written as [21] $\gamma \sim\left(\eta / R_{1}^{2}\right)\left(\phi_{2} / 1\right.$ $\left.-\phi_{2}\right)$. Note that this expression yields correct limiting behaviors for $\phi_{2} \rightarrow 0$ and $\phi_{2} \rightarrow 1$. It is valid for low salt concentrations, since it does not consider that the hydrodynamic interactions between beads are screened by the counterions in the presence of an external electric field [24]. Taking all these facts into account permits the calculation of $\mu^{*}$ and assures the fact that it depends linearly on $Z_{\text {eff }}$.

The electrophoretic mobility measurements for different $Z$ 's are presented in Table I. As can be seen, $\mu$ passes through a minimum as $Z$ increases [25]. This behavior is a result of the competing decrease of charge density and

TABLE I. Polymer network charge and electrophoretic mobility measurements. The errors in $\mu$ are $\sim 0.15 \times 10^{-8} \mathrm{~m}^{2} \mathrm{~V}^{-1} \mathrm{~s}^{-1}$. As $Z$ increases, the particle swells from $\sim 230 \mathrm{~nm}$ to $\sim 1200 \mathrm{~nm}[5]$.

\begin{tabular}{cc}
\hline \hline$\left(10^{-6}\right) \mathrm{Z}$ & $\mu\left(10^{-8} \mathrm{~m}^{2} \mathrm{~V}^{-1} \mathrm{~s}^{-1}\right)$ \\
\hline 1.75 & 3.72 \\
2.63 & 3.53 \\
3.61 & 3.33 \\
5.32 & 3.17 \\
6.02 & 3.35 \\
6.89 & 3.54 \\
7.35 & 3.71 \\
7.83 & 3.83 \\
\hline
\end{tabular}


polymer-solvent friction as the network swells. The charge density reduction (remember that even though $Z$ increases, the particle volume also increases because of swelling) tends to make $\mu$ decrease, while the decrease in $\gamma$ leads to its increase. The experimental results show that for smaller $Z$ 's the electrophoretic mobility is controlled by the charge density, while for larger Z's it is controlled by the friction [21].

The experimental measurements of the electrophoretic mobility combined with the theoretical calculation of $\mu_{s}$ lead to the value of $\mu^{*}$. The dependence of $\mu^{*}$ on the bare charge is presented in Fig. 5. As can be seen, $\mu^{*}$ scales as $Z^{0.5}$. This asymptotic behavior is in perfect agreement with the theoretical prediction $Z_{\text {eff }} \sim Z^{0.5}$, since $\mu^{*}$ depends linearly on $Z_{\text {eff }}$, according to Eq. (22).

We have presented a thermodynamic theory for suspensions of ionic microgel particles. The state of this system can be characterized by a diameter and an effective charge of the particles. It was found that microgels with a large number of ionized groups exist in a swollen state in which their effective charge scales as $Z_{\text {eff }} \sim Z^{0.5}$. This asymptotic behavior has been verified experimentally, showing that the electrophoretic mobility is, indeed, controlled by the renormalized bulk charge.

\section{ACKNOWLEDGMENTS}

This work was supported in part by CNPq (Brazil) and Comisión Interministerial de Ciencia y Tecnología (Spain) under the Projects No. MAT2000-1550-C03-02 and No. MAT2001-2767.
[1] P.J. Flory, Principles of Polymer Chemistry (Cornell University Press, Ithaca, NY, 1953).

[2] A. Katchalsky, J. Polymer J. Polym. Sci. 7, 393 (1951).

[3] F.E. Harris and S.A. Rice, J. Phys. Chem. 58, 725 (1954).

[4] K. Kajiwara and S.B. Rossmurphy, Nature (London) 355, 208 (1992).

[5] A. Fernández-Nieves, A. Fernández-Barbero, B. Vincent, and F.J. de las Nieves, Macromolecules 33, 2114 (2000).

[6] O. Quadrat and J. Snuparek, Prog. Org. Coat. 18, 207 (1990).

[7] N.A. Peppas, Curr. Opin. Colloid Interface Sci. 2, 531 (1997).

[8] C.N. Likos, Phys. Rep. 348, 267 (2001).

[9] O.V. Borisov and E.B. Zhulina, Eur. Phys. J. B 4, 205 (1998).

[10] I. Sogami and N. Ise, J. Chem. Phys. 81, 6320 (1984).

[11] R. van Roij and J.P. Hansen, Phys. Rev. Lett. 79, 3082 (1997); R. van Roij, M. Dijkstra, and J.P. Hansen, Phys. Rev. E 59, 2010 (1999).

[12] Y. Levin, M.C. Barbosa and M.N. Tamashiro, Europhys. Lett. 41, 123 (1998); M.N. Tamashiro, Y. Levin, and M.C. Barbosa, Physica A 258, 341 (1998).

[13] J.P. Hansen and H. Löwen, Annu. Rev. Phys. Chem. 51, 209 (2000).

[14] P.B. Warren, J. Chem. Phys. 112, 4683 (2000).
[15] A. Diehl, M.C. Barbosa, and Y. Levin, Europhys. Lett. 53, 86 (2001).

[16] S. Alexander, P.M. Chaikin, P. Grant, G.J. Morales, P. Pincus, and D. Hone, J. Chem. Phys. 80, 5776 (1984).

[17] H. von Grueneberg and R. von Roij, Europhys. Lett. 55, 580 (2001).

[18] P. Linse and V. Lobaskin, Phys. Rev. Lett. 83, 4208 (1999); J. Chem. Phys. 112, 3917 (2000).

[19] A. Loxley, and B. Vincent, Colloid Polym. Sci. 275, 1108 (1997).

[20] D.D. Perrin, Dissociation Constants of Organic Bases in Aqueous Solution (Butterworths, London, 1965).

[21] A. Fernández-Nieves, A. Fernández-Barbero, B. Vincent, and F. J de las Nieves, J. Phys.: Condens. Matter 12, 3605 (2000).

[22] H.C. Brinkman, Res. Q. 2, 190 (1949); , Proc. R. Acad. Sci. Amsterdam 50, 618 (1947).

[23] M.A. Cohen Stuart, F.H.W.H. Waajen, T. Cosgrove, B. Vincent, and T. Crowly, Macromolecules 17, 1825 (1984).

[24] D. Long, J-L. Viovy, and A. Ajdari, Phys. Rev. Lett. 76, 3858 (1996).

[25] S. Hirotsu, Phase Transitions 47, 183 (1994). 